\title{
Waveguide solar concentrator design with spectrally separated light
}

\author{
Céline Michel $^{\mathrm{a}}$, Pascal Blain ${ }^{\mathrm{a}}$, Lionel Clermont ${ }^{\mathrm{a}}$, Fabian Languy ${ }^{\mathrm{a}}$, Cédric Lenaerts ${ }^{\mathrm{a}}$, \\ Karl Fleury-Frenette ${ }^{\mathrm{a}}$, Marc Décultot ${ }^{\mathrm{b}}$, Serge Habraken ${ }^{\mathrm{b}}$, Denis Vandormael ${ }^{\mathrm{c}}$, Rudi Cloots ${ }^{\mathrm{d}}$, \\ Gopala Krishna V.V. Thalluri ${ }^{\mathrm{d}}$, Catherine Henrist ${ }^{\mathrm{d}}$, Pierre Colson ${ }^{\mathrm{d}}$, Jérôme Loicq ${ }^{\mathrm{a}, *}$

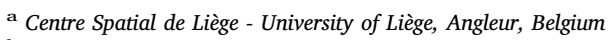 \\ ${ }^{\mathrm{b}}$ Hololab, Science Faculty, University of Liège, Liège, Belgium \\ c Sirris, Liège Science Park, Seraing, Belgium \\ d Group of Research in Energy and ENvironment from MATerials (GREENMAT), University of Liège, Liège, Belgium
}

\section{A R T I C L E I N F O}

\section{Keywords:}

Solar concentrator

Light splitting

Waveguiding

Nonimaging optics

Fresnel lens

Diffraction grating

\begin{abstract}
A B S T R A C T
In this article, we propose a new solar concentrator based on spectral splitting of sunlight. Spectral splitting has the objective to collect different spectra onto spectrally adapted solar cells for a more efficient use of the Sun's spectrum. Its combination with solar concentration makes an alternative to classical technologies. The proposed concentrator is composed of a diffractive/refractive optical element that spectrally splits and focuses the light onto a waveguide. The light is then conducted by total internal reflection towards the two specific solar cells. The optical concept and optimization of each element is presented in this paper. An adaptation for dye sensitized solar cells is performed. A geometrical factor around $5 \times$ is reached. Finally, theoretical optical efficiency, the manufacturing process and experimental testing with a collimated Sun simulator are presented.
\end{abstract}

\section{Introduction}

For many years now, much effort has taken place in the quest for new ways to reduce the production costs of electricity generated by photovoltaic processes. The concept of solar concentration has been of particular interest in this context. The basic principle of solar concentration is to use a low cost optical component that concentrates sunlight onto photovoltaic (PV) cells (Luque and Andreev, 2007; McConnell and Symko-Davies, 2006; O'Gallagher, 2008; Takamoto et al., 2005; Wurfel, 2005). Three main families of concepts exist in relation to photovoltaic processes: reflective optics (Chong et al., 2009; Coventry, 2005; Cvetković et al., 2007; Feuermann and Gordon, 2001), refractive optics (Leutz, 2000; Vasylyev, 2005; Xiea et al., 2011; FerrerRodrguez et al., 2017; Steiner et al., 2016; Helmers et al., 2013) and light guide techniques (Gallagher et al., 2007; Goldschmidt et al., 2009a; Goldschmidt et al., 2009b; Karp et al., 2010).

In refractive optics a sub division is made by the addition of diffractive component as surface relief grating and holographic elements (Blain et al., 2014; Michel et al., 2014) to correct the chromatic aberration of the system or to split light into different wavelength components (Michel et al., 2015).

In this paper we focus on concentrator based on waveguiding. A number of optical designs, experimental prototype and commercial system have been proposed. One of the first companies proposing a commercial concept is Morgan solar (Pindar et al., 1992). Moreover a number of complementary works have been performed with innovative concepts. As the main objective for concentrator waveguide is to redirect sun-light to PV cells placed on its edges. The strategies to convert incident angles to total internal reflection (TIR) allowing light propagation through the waveguide are various. Lets cite: Tapered concentrator (Park et al., 2010), backlight concentrator (Ming-Chin et al., 2009), luminescent concentrator (Goldschmidt et al., 2009b; Li et al., 2016; van Sark et al., 2008) and micro-optics waveguided concentrator (Karp et al., 2011; Peng et al., 2014).

Multi-junction (MJ) cells are often used in association with concentrators due to their high conversion efficiency. Their high cost is offset by the concentration ratio. Nevertheless, it is of great interest to find other solutions, further reducing the cost of PV modules. One way is the use of other types of cells, combined in different configurations. Spectrally and spatially (Vlasov et al., 2013; Mojiri et al., 2013) splitting incoming light is a solution. It allows different spectra to reach different cells that are spatially separated and thus independent, widening the combination possibilities compared to MJ cells. This physical separation also allows an electrical independence of the cells. The cell sensitivity to incident spectrum variations is then reduced compared to MJ cells (which are connected in series by definition).

\footnotetext{
* Corresponding author.

E-mail address: j.loicq@ulg.ac.be (J. Loicq).
} 
With a spectrum splitting solar concentrator, it has been shown (Zhao, 2011) that the performance of lower efficiency cells integrated over a year gives a very positive budget.

The concept presented in this paper is based micro-optics elements as micro-prisms associated with surface relief grating to allow spectral splitting. Spectral splitting (Fixler et al., 2011; Fisher and Biddle, 2011; Stefancich et al., 2012) enables the operation of single-junction devices to operate within the energy range where they best perform. Because cells operate independently, they can be designed and optimized separately. Obviously the concept of spectral splitting is very versatile and can be adapted to any wavelength range.

The study presented is based on a focusing element combined with a planar waveguide (in order to further increase the concentration ratio), coupled with spectral splitting. Thereby, two different cells not easily compatible with the classical concept of MJ cells can be combined. Since cell costs should remain low, the developed concentrator can be advantageous even with low concentration. The aim is to reach around $5 \times$ solar concentration. This low solar concentration limits working cell temperatures, which is a great advantage since cell efficiency is known to decrease with increasing temperature.

The concept has been introduced in a previous paper (Blain et al., 2014), applied to two dye sensitized solar cells (DSSC). Here, we will expose the main characteristics and optical performances achievable by this concentrator concept, and further describe each optimization and development step: calculation and optimization methodology, including cell characteristics used for the design, and optimization of each optical element both individually and then together, to reach the best optical performances. Experimental laboratory results on optical properties conclude this work.

\section{Concentrator concept}

Beyond sunlight concentration, the main objective of the presented waveguide concentrator is to divide the solar spectrum into two distinct parts. These two spectral bands correspond to the respective solar cell sensitivities chosen for the cells. Each cell is connected to the waveguide at its opposite edges. The concentrator is composed of two main entities, depicted in Fig. 1:

- A focusing lens array combined with a diffraction grating.

- A waveguide with engraved microstructures (named V-grooves).

The focusing array and diffractive grating combination is designed to focus light and perform a spectral broadening on the microstructures

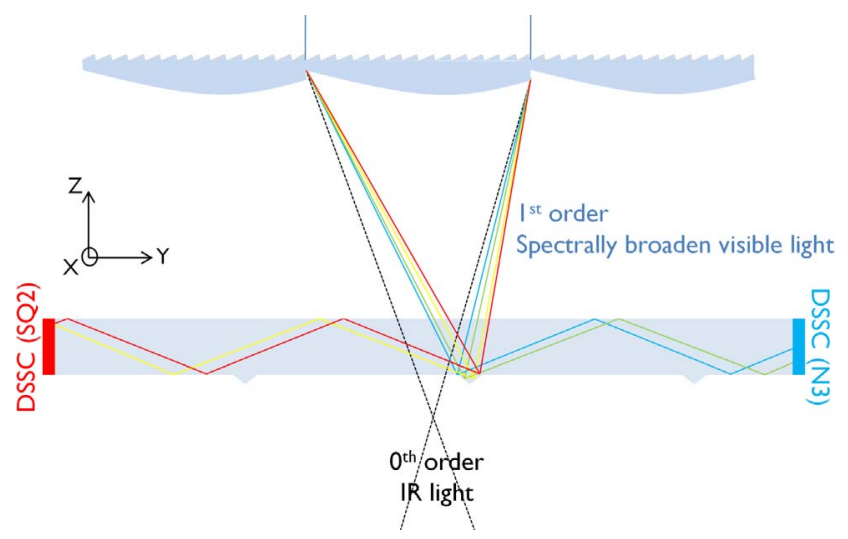

Fig. 1. General concept of the concentrator. It includes two main optical elements: a grating/lens combination, coupled to a waveguide. The grooves of the waveguide, placed in the focal plane of each grating/lens element, split the incident light (spectrally dispersed by the grating/lens combination) to redirect it towards specific cells at the edges of the waveguide. This concentrator concept simultaneously concentrates and spectrally splits incident light.

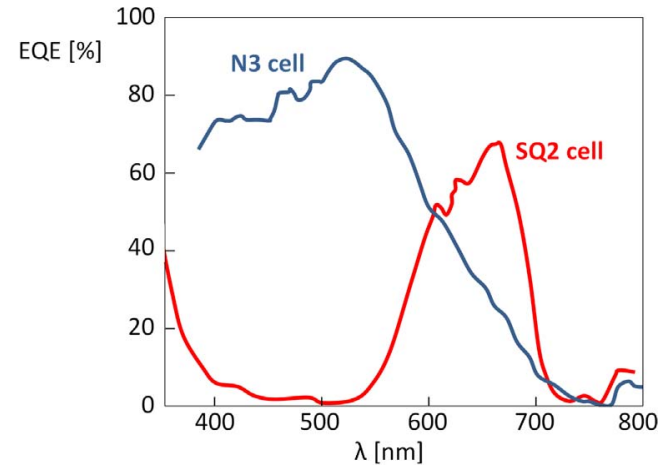

Fig. 2. External quantum efficiencies (Thalluri et al., 2013) of the N3 dye-sensitized solar cell (blue curve) and the SQ2 dye-sensitized solar cell (red curve) (Thalluri et al., 2013, 2015). (For interpretation of the references to color in this figure legend, the reader is referred to the web version of this article.)

placed on the back side of the waveguide, as depicted on Fig. 1. These microstructures reflect light so that the light is in total internal reflection along the whole waveguide. The light is then guided to the edges of the waveguide. On those edges, light flux is composed of different spectrum components respectively tuned to correspond to the sensitivity of the assembled cells (see Section 3). The grating and the focusing lens combined with the position and the shape of the V-groove give different degrees of freedom in order to adapt and optimize the system. The concentration ratio is given by the ratio of total lens surface and total cell surface.

\section{Cell spectral properties}

Two DSSC dyes were selected for this study: dyes N3 and SQ2. Spectral solar cell properties used for the optical design come from GREENMAT $^{\mathrm{d}}$, according to previous published works (Thalluri et al., 2013, 2015), briefly described in Section 6.1. Respectively, these dyes have high external quantum efficiencies (EQEs) in spectral a band-pass from $400 \mathrm{~nm}$ to $600 \mathrm{~nm}$ and $600 \mathrm{~nm}$ to $700 \mathrm{~nm}$, as depicted in Fig. 2. From our concentrator point of view, these optimal spectral bands define the ideal splitting wavelength at about $600 \mathrm{~nm}$.

\section{Material optical properties}

To be cost effective, the concentrator design has to be compatible with large scale mass production and industrial manufacturing processes. Hence, each optical element that constitutes the concentrator will be manufactured with thermoplastic material. A careful study of potential adapted materials for refractive concentrators, compliant with an injection molding process, has been realized by Sirris ${ }^{\mathrm{c}}$. This leads to the choice of a specific Polymethyl Methacrylate (PMMA): Plexiglas ${ }^{\circledast}$ Solar IM20 from Evonik (Evonik Performance Materials GmbH, 2016), due to its high transmittance and resistance to yellowing under sunlight. Transmission curves have been measured at CSL. Results are depicted in Fig. 3.

The refractive index of this PMMA was measured at Centre Spatial de Liège ${ }^{\mathrm{a}}$ (CSL) by refractometry (Abbemat WR-MW refractometer (Anton Paar, 2016)). It can be described by the following equation (Languy et al., 2009):

$n(\lambda)=\sqrt{A+\frac{B 1 \cdot \lambda^{2}}{\lambda^{2}+B 2}+\frac{C 1 \cdot \lambda^{2}}{\lambda^{2}+C 2}+\frac{D 1 \cdot \lambda^{2}}{\lambda^{2}+D 2}}$

with the fitting parameters listed in Table 1 .

The refractive index is plotted in Fig. 4. Since this PMMA has very low absorption in the visible spectrum, it is assumed to have no absorption for our simulations.

Another important material is the glue used to assemble the DSSC 


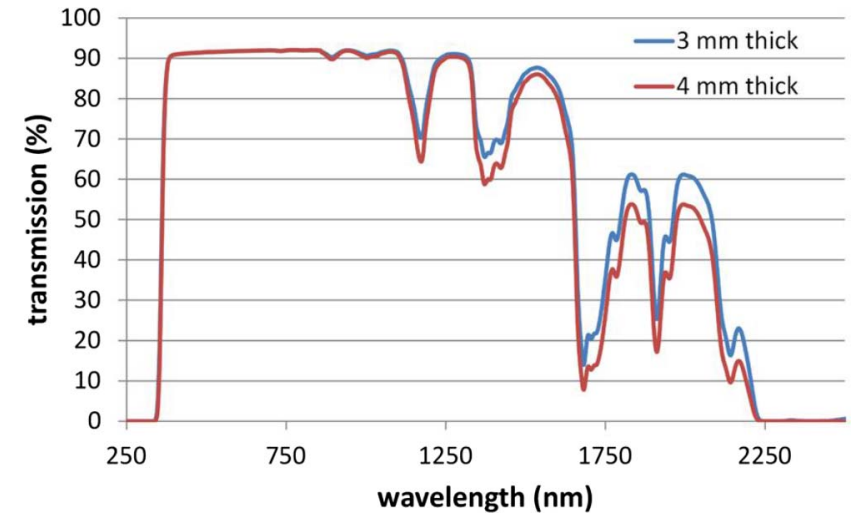

Fig. 3. Transmission curve of PMMA (Plexiglas ${ }^{\circledast}$ Solar IM20 from Evonik) measured at CSL.

Table 1

Fitting parameters corresponding to Eq. (1), with $\lambda$ expressed in $[\mu \mathrm{m}]$.

\begin{tabular}{cccc}
\hline $\mathrm{A}$ & 1.026 & $\mathrm{C} 2$ & -0.001597 \\
$\mathrm{~B} 1$ & 0.6972 & $\mathrm{D} 1$ & 0.2037 \\
$\mathrm{~B} 2$ & -0.001529 & $\mathrm{D} 2$ & -0.04259 \\
$\mathrm{C} 1$ & 0.2722 & & \\
\hline
\end{tabular}

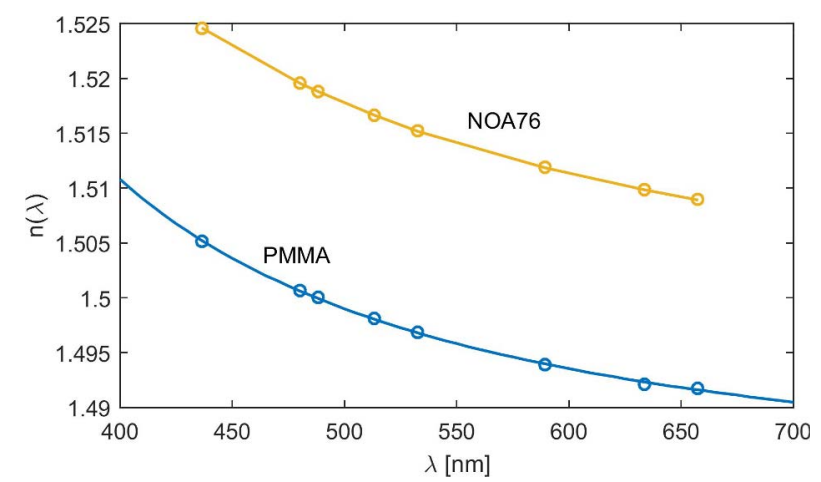

Fig. 4. Refractive index of the NOA76 (yellow) and the chosen PMMA (Plexiglas ${ }^{\circledast}$ Solar IM20 from Evonik) (blue), extracted from experimental refractometry measurements performed at CSL (circles) and Sellmeier approximation (line). (For interpretation of the references to color in this figure legend, the reader is referred to the web version of this article.)

and waveguide. This material is also used to imprint the grating on PMMA lenses. Criteria are its adhesion with glass and PMMA, transparency, viscosity and refractive index. The chosen material is Norland Optical Adhesive 76 (NOA76) glue (Norland Products, 2016). The NOA76 refractive index is very close to PMMA, avoiding additional losses at interfaces. The refractive index has been characterized at CSL, results are shown in Fig. 4.

\section{Optical components: Simulations and optimization methods}

The main new aspect of this concentrator is the combination of the diffractive, refractive and waveguiding optical components, to reach an efficient spectral separation maintaining high optical efficiency. The optical optimization was thus a large part of the global development. The first optimization process is performed on the focusing element alone, i.e. the grating combined with the lens, in Section 5.1. A second optimization process is then performed considering the combination with the waveguide, presented in Section 5.2.

After optimization, theoretical performance and tolerances have been computed, described in Section 5.4. Finally, these results are compared with the performance of an experimental prototype in Section 6.
Hypotheses and means for optical simulations and optimizations are the following:

- Final simulations were performed with the non-sequential ray-tracing software ASAP ${ }^{\circledR}$. First step optimization developments are performed with in-house ray-tracing Matlab ${ }^{\circledR}$ scripts.

- Optical elements (grating/lens and waveguide) are in PMMA whose properties are described in Section 4.

- The engraved microstructures of the waveguide are covered by a reflective coating.

- Fresnel reflections are taken into account, but scattering effects due to roughness are ignored.

- The source has a semi-field of $16^{\prime}$ corresponding to sunlight divergence. The AM1.5D solar spectrum is used (National Renewable Energy Laboratory, 2015).

The global efficiency of the system corresponds to the sum of the fluxes on detector 1 and on detector 2, considering the respective spectral domains (i.e. $400-600 \mathrm{~nm}$ and $600-700 \mathrm{~nm}$ ). The global efficiency is normalized by the input flux on the $400-700 \mathrm{~nm}$ spectral domain of the AM1.5 solar spectrum.

\subsection{Focusing component}

The first stage of the concentrator is composed of a grating and a lens. The objective of such elements combined is to create a spectrally spread focal spot on the bottom side of the waveguide.

\subsubsection{The grating}

Many configurations using different diffraction orders and grating types have been studied, to reach the best compromise for our application. In our case, the most compliant grating is a surface relief blazed grating, working in transmission, maximizing the diffraction efficiency of 1st diffraction order in the visible spectrum, and compatible with large scale and low cost production.

The diffraction efficiency of such a grating is depicted in Fig. 5. The maximum efficiency position for 1st diffraction order depends on the chosen blaze wavelength $\left(\lambda_{\text {blaze }}\right)$.

The grating geometrical behavior is described by the grating Bragg equation:

$n_{1} \sin \left(\theta_{\text {diff }}\right)=n_{3} \sin \left(\theta_{\text {in }}\right)-\frac{m \cdot \lambda}{\Lambda}$

with $n_{1 / 3}$ respectively the refractive indices of incident and exit spectra (air in our case), $\theta_{\text {in }}$ the incidence angle, $m$ the diffraction order, $\lambda$ the wavelength, $\Lambda$ the grating period, and $\theta_{\text {diff }}$ the diffraction angle. The first diffraction order is then spectrally dispersed. It can thus be split on

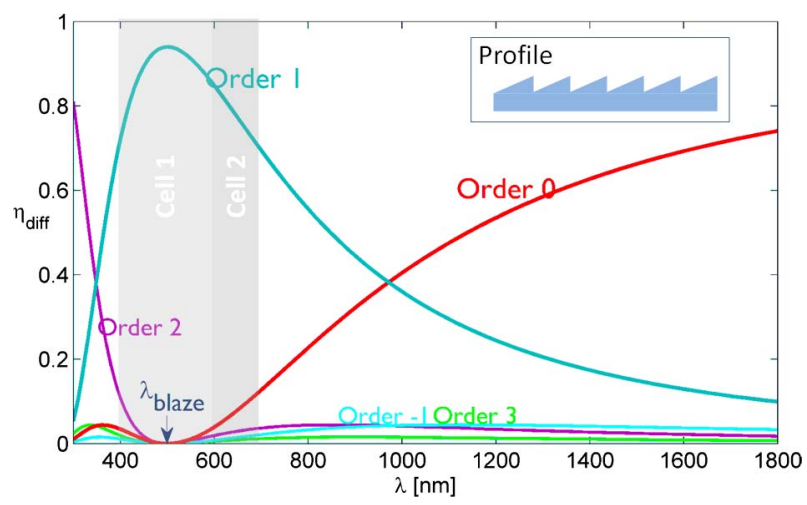

Fig. 5. Diffraction efficiencies of a surface relief blazed grating, with a $\lambda_{\text {blaze }}$ equal to $500 \mathrm{~nm}$ : the main part of visible light between 400 and $700 \mathrm{~nm}$ convertible by DSSCs (grey areas) is diffracted in the 1st diffraction order. An example of the profile of a blazed grating (not to scale) is depicted in the top right corner. 
the focal plane (by the grooves of the waveguide) into two spectrally different parts. Non-diffracted light (0th order diffracted light), containing mainly IR light, misses the grooves and goes through the waveguide without interacting, as depicted in Fig. 1. Since this spectral domain is not convertible by the DSSCs, this configuration avoids heating of cells due to its absorption.

From Eq. (2), the larger the period, the smaller the diffraction angle, and at the end, the narrower the spectral broadening. The choice of an adequate grating period from a geometrical point of view will be discussed in combination with the lens in Section 5.1.3. Nevertheless, for diffraction efficiency, it is better to use large grating periods, avoiding shadowing (Swanson, 1989) encountered for small periods $(<20 \mu \mathrm{m})$, and allowing grating simulation with the scalar diffraction theory (Goodman, 1968).

To be in the scalar diffraction theory regime in interesting for simulations since it allows the decoupling of grating parameters: diffraction efficiencies are directly linked to the blaze wavelength (Swanson, 1989), while the period drives only the diffraction angles (Goodman, 1968) through Eq. (2). The two parameters can thus be optimized independently: the period is defined according to the lens shape and desired spectral broadening, while the blaze wavelength is determined to collect in 1st diffraction order the largest part of the sun spectrum that can be converted by both cells. This wavelength will be optimized after the global geometry is established, to take into account the exact spectral separation balance.

\subsubsection{The lens}

The method of lens design is implemented to minimize the focal spot size of first order diffracted light due to decoupling effects in the waveguide. Indeed, as will be shown in Section 5.2.3.2, the decoupling is proportional to the size of the microstructured groove. But, at the same time, a large spectral dispersion is needed in the focal plane, defining the splitting efficiency. The most restrictive consideration is nevertheless decoupling, and thus focusing quality. Hence, the nonimaging method used to design the lens is that developed in Michel et al. (2014) for a similar concentrator for space applications, without waveguide. The lens shape is defined by four main parameters: its $F \sharp$, its off-axis, the spectra to be collected by respective cells, and the grating period. The off-axis is the distance between the geometrical center of the lens and the center of the focal spot of the first diffraction order. All parameters are closely linked.

The choice of parameter combinations is differently constrained for our concentrator than in the reference study (Michel et al., 2014). Considerations leading to the best combination are described in the following sections. Final conclusions on optimal parameter combinations will be addressed in Section 5.3.

\subsubsection{The grating/lens combination}

For the optimization of the grating/lens combination, two objectives are followed:

1. Minimizing the size of focal spots to reduce decoupling effects due to the contiguous grooves in the waveguide.

2. Maximizing the spectral dispersion of the first order diffracted light on the focal plane to increase the global spectral splitting efficiency.

Dispersion and size considerations are detailed in the following two subsections.

5.1.3.1. Size considerations. The size of the focal spot is defined as the diameter of the spot collecting all the rays coming from the first diffracted order in the spectral range, between the first and the last wavelength convertible by the combined two cells. It depends on the three following parameters: $F \sharp$, off-axis and period. For a fixed $F \sharp$, the size is plotted according to the off-axis and the period in Fig. 6. A large interdependence between these parameters is observable, leading to a

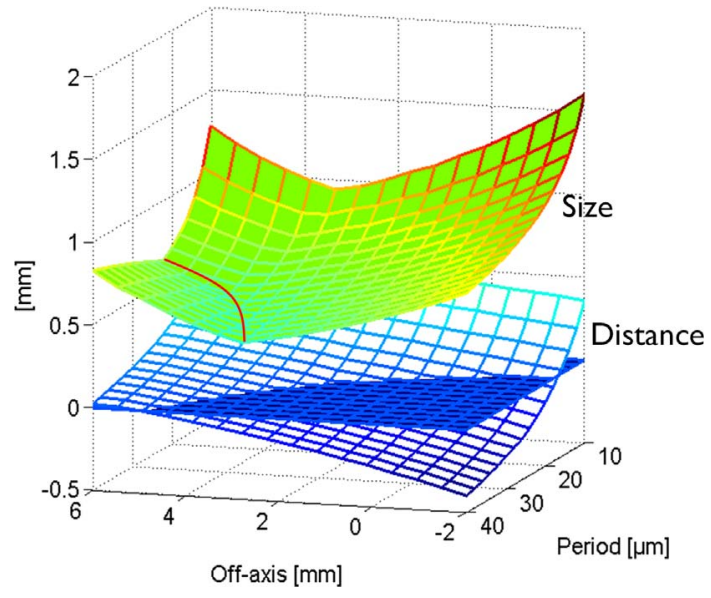

Fig. 6. Spot size dependence with off-axis and period combinations. The overlapping zone must be excluded to avoid IR light reaching PV cells.

minimum dimension for a particular combination. The overlapping zone (distance $<0$ ) between the 1 st and 0 th orders is to be excluded in order to avoid IR non-diffracted light reaching the PV cells. Some combinations of parameters are thus not recommended, such as small off-axis combined with large grating period.

Drawing the same data for other $F \sharp$ leads to an optimum $F \sharp$ around 2 , allowing the minimum size of focal spots. The final $F \sharp$ will nevertheless be chosen later, after waveguiding consideration.

5.1.3.2. Dispersion considerations. The light dispersion in the focal plane is defined by the parameter $D$ (illustrated in Fig. 7). $D$ is the area of spectral overlapping in the focal plane around $\lambda_{\text {division }}$ defining the optimum separation of cell EQE spectra. In Section $3, \lambda_{\text {division }}$ was fixed at $600 \mathrm{~nm}$. So, the smaller $D$ is, the larger the dispersion.

As for focal spot size considerations, the dependence of the dispersion parameter $D$ has been plotted in Fig. 8 according to period, off-axis, and for several $F \sharp$. The conclusion (for the grating/lens combination alone) is straightforward since the light dispersion is inversely proportional to the grating period: the best solution is to use the minimum grating period. The off-axis influence decreases for large $F \sharp$. Optimum parameters have nevertheless to include all optical elements of the concentrator, even waveguiding.

\subsection{Waveguide design and light coupling}

The second step of the system optimization corresponds to the coupling of the focusing element with the waveguide and its microstructures. The V-grooves are placed on the waveguide with the same periodicity as the lenses, respectively in their focal plane (corrected by the PMMA thickness of the waveguide), as depicted in Fig. 1. These microstructures follow two objectives: the first is to redirect the light into total internal reflection conditions and the second is to make the spectral separation, i.e. select the wavelength range associated with the cells and send it to them. Groove shapes and positioning have thus to be optimized, which is described in following sections.

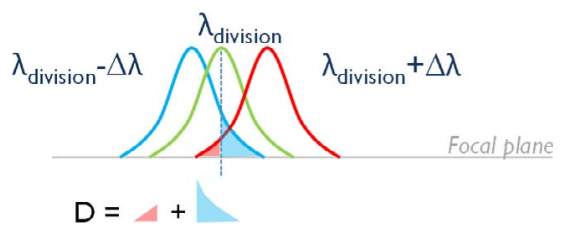

Fig. 7. Principle for the definition of the optimization parameter $D$ : area of spectral overlapping in the focal plane around the chosen splitting wavelength $\left(\lambda_{\text {division }}\right.$, previously fixed at $600 \mathrm{~nm}$ ). $D$ is linked to the dispersion and then to the spectral splitting efficiency. 


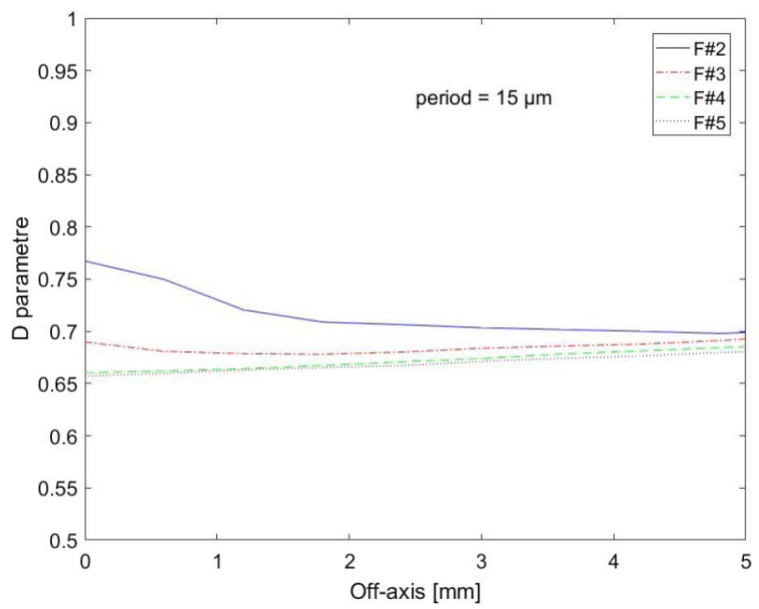

(a)

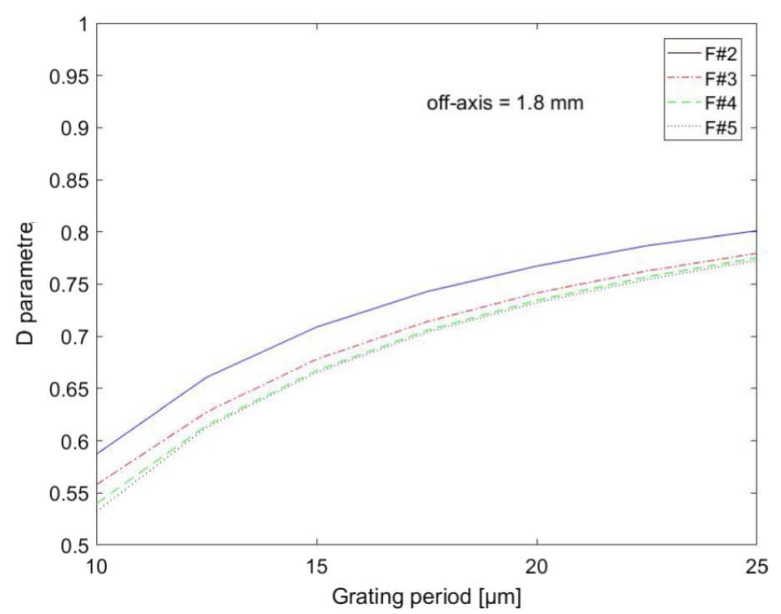

(b)

Fig. 8. Parameter $D$ dependence with off-axis (left) and grating period (right), for several $F \sharp$.

Waveguiding induces optical losses by absorption and mainly by decoupling. In the optimization process, effects of this decoupling on the performance of the planar concentrator were investigated.

\subsubsection{Groove peak positioning: Spectral splitting efficiency}

The groove causes the spectral separation (see Fig. 1). Since cells have specific EQEs with high conversion efficiency in different spectra, the separation has to occur at the transition between them. In our case, according to Section 3, the middle wavelength is $600 \mathrm{~nm}$. Due to the lens and sun divergence, there is an overlap between wavelengths in the focal plane, described by the dispersion parameter (see Fig. 7). Hence, the center of the groove for the spectral separation is positioned at the maximal intensity of focused light with $600 \mathrm{~nm}$ wavelength.

The focal spot asymmetry around the intensity peak at $600 \mathrm{~nm}$ has been considered for groove design.

\subsubsection{Groove inclination: Total internal reflection condition}

The optimal groove angle with respect to the horizontal is $30^{\circ}$. This has been chosen so that the reflected rays (in $400-700 \mathrm{~nm}$ spectral range) stay in total internal reflection (TIR) in the waveguide, until reaching a cell or decoupling.

\subsubsection{Optical losses by decoupling}

Decoupling occurs when a guided ray hits another V-groove microstructure before reaching the PV cell. In that case, the ray is sent to a direction which, most of the time, is not in TIR. Consequently, this ray is lost. The decoupling thus acts directly on the efficiency of the concentrator. All design parameters have an influence, and have to be chosen carefully to avoid large optical losses in the waveguide.

5.2.3.1. F-number. Fig. 9 presents the global efficiency of a 5 groove system according to the F\#. From this Figure, it is clearly shown that small $\mathrm{F} \sharp$ give better results. This is due to the fact that a small $\mathrm{F} \sharp$ provides a larger angular range than a high $\mathrm{F} \sharp$ : the wider the angular range, the more likely a ray will hit another groove, but with a smaller impact in terms of energy lost. Conversely, the narrower the angular range, the more chance all the energy will be lost on a groove because all rays hit the groove at the same time. Thus, from a decoupling point of view, it is preferable to use lenses with an $\mathrm{F} \sharp$ as small as possible.

5.2.3.2. Groove width. Obviously, groove size has an impact on the flux and on the spectrum. It cannot be smaller than the focal spot size to collect all 1st order diffracted light that can be converted by the PV cells. On the other hand, oversizing the groove will increase decoupling. This effect is depicted in Fig. 10. This figure shows the evolution of the

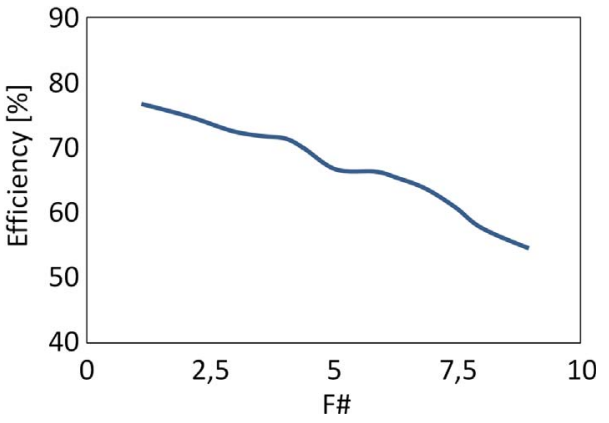

Fig. 9. System global efficiency according to the $F \#$ : a smaller $F \#$ gives rise to a wider angular dispersion, and then on average to lower optical losses by decoupling. Simulation for 5 grooves, $5 \mathrm{~mm}$ thick waveguide.

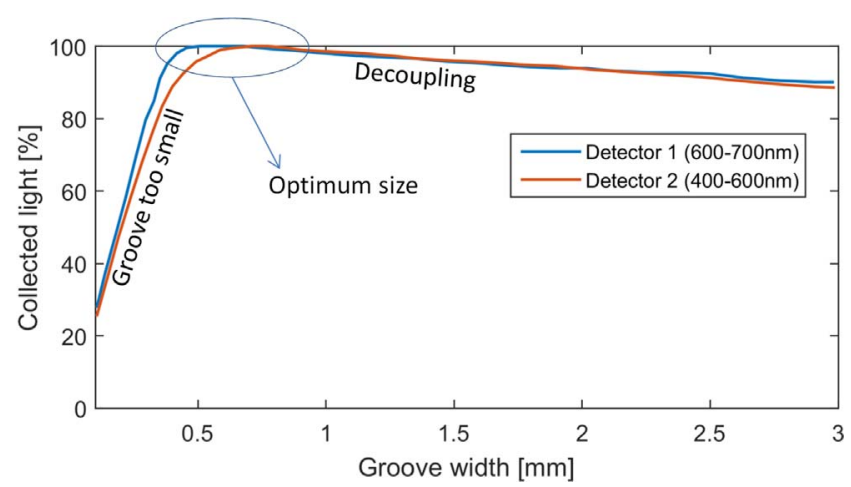

Fig. 10. Fraction of incident flux reaching each detector according to the groove width. A too small groove does not collect all light of interest, a too large groove induces additional losses by decoupling (example here for 5 grooves, $5 \mathrm{~mm}$ thick waveguide and $\mathrm{F} \#=2$ ).

flux reaching each individual detector, integrated over their spectral domain of interest and normalized, as a function of the groove size. On both detectors, the flux presents a maximum when the groove width corresponds to the focal spot size previously determined, large enough to collect all light of interest. Oversizing induces additional decoupling. Nevertheless, oversizing can be permitted for manufacturing tolerance considerations (see Section 5.4.2).

5.2.3.3. Waveguide thickness and groove number. As with large groove size, a larger number of grooves (identical to the number of lenses) induces losses by decoupling. These losses also depend on the waveguide thickness, defining the number of reflections in the 


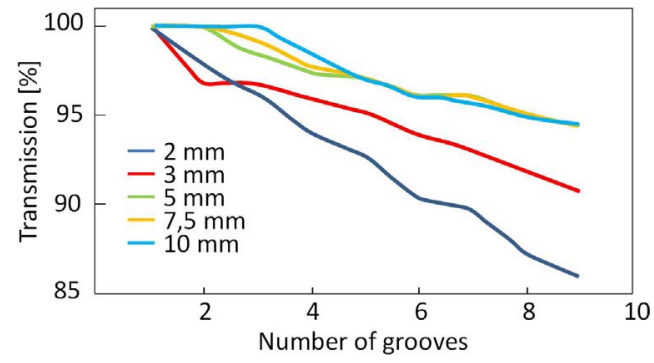

Fig. 11. Waveguide transmission evolution according to the number of grooves $(2 \mathrm{~mm}$ wide), for different waveguide thicknesses (2, 3, 5, 7.5 and $10 \mathrm{~mm}$ thick). $F \sharp=2$.

waveguide and thus the probability of decoupling. Fig. 11 depicts an example of transmission evolution according to the number of grooves for different waveguide thicknesses.

\subsection{Compromise and final configuration}

Optical efficiency of the lens and waveguide combination is influenced by the parameters described in the previous sections. Each decision for the optimum combination is detailed hereunder:

1. $F \#$ : To avoid large optical losses due to decoupling, a small $F \#$ is preferable (see Section 5.2.3.1). However, from a manufacturing point of view and to avoid optical losses by shadowing of the teeth, it is preferable to use lenses with $F \sharp$ not lower than 2 (Davis, 2009). This is why $F \#=2$ has been chosen.

2. Off-axis: With an $\mathrm{F} \sharp$ of about 2 , following the graph in Fig. 8, the offaxis of the lens has to be between 2 and $5 \mathrm{~mm}$, in order to maximize the dispersion. However, coupling with the waveguide induces some limitation on the maximum off-axis. A too large off-axis creates asymmetry in focusing beam with large incident angles: combined with groove inclination, vignetting and thus optical losses appear. The best compromise is found for an off-axis around $1.1 \mathrm{~mm}$.

3. Waveguide thickness and groove number: As seen in Fig. 11, optical losses due to decoupling are smaller for thicker waveguides. Our prototype will include 5 focusing elements, i.e. 5 grooves on the waveguide. The waveguide thickness is then fixed to $5 \mathrm{~mm}$, a compromise between decoupling losses, absorption losses and concentration ratio (linked to cell area and thus to the waveguide thickness).

4. Grating/lens - waveguide distance: In order to get an optimal separation of the two spectral domains, the central field beam at the separation wavelength $(600 \mathrm{~nm})$ must converge on the center of the groove. But the transmission from air to PMMA modifies the light path. So, the appropriate lens-waveguide distance and the lateral position of the grooves must be adapted, taking into account the refractive index of the waveguide and its thickness. The optimal distance between the upper face of the waveguide and the back face of the lens is $18 \mathrm{~mm}$ in our case, for a lens with $10 \mathrm{~mm}$ width and an $\mathrm{F} \sharp=2$.

5. Grating period: From a dispersion point of view, as was detailed in Section 5.1.3.2 a small period maximizes spectral splitting efficiency due to a larger spectral dispersion in the focal plane. This effect is presented in Fig. 12(a), depicting the normalized fraction of light collected on each facet of the groove, according to the wavelength: spectral splitting efficiency is larger for smaller grating periods.

However, smaller grating periods lead to larger focal spot sizes (see Fig. 6), and then to larger groove dimensions. Since groove dimensions are closely linked to decoupling losses, the smaller the period is, the larger the optical losses due to decoupling, as depicted in Fig. 10. The global concentrator optical transmission efficiency is depicted in Fig. 12(b). Finally, this means that a compromise has to be found between small and large periods. The best compromise seems to converge around $20-25 \mu \mathrm{m}$ : maximizing spectral splitting (Fig. 12 (a)) while limiting optical efficiency losses (Fig. 12 (b)).

6. Groove width: The groove width has to be defined taking into account decoupling effects (see Fig. 10), and collection efficiency to redirect light in total internal reflection in the waveguide. But it has also to integrate some tolerance in order to avoid losses due to fabrication errors. Finally, a groove width of $2 \mathrm{~mm}$ has been chosen.

7. Groove shape: The grooves will be symmetrical, with their peak placed at the maximum of the $600 \mathrm{~nm}$ light focalization point, and $30^{\circ}$ facet inclination.

8. $\lambda_{\text {blaze }}:$ As previously introduced, the blaze wavelength of the diffraction grating has an important impact on the performance of the concentrator since it defines the energy balance between the zero and first diffraction orders. For our concentrator, first order diffracted light has to be maximal in the spectral range convertible by the PV cells. Moreover, to maximize global electrical output power, solar spectrum has to be included in optimization, as well as optical losses in the concentrator. When all geometrical aspects are fixed, the fraction of light reaching each cell is known, according to the wavelength. In Fig. 13, the global efficiency is presented over the two spectral bands together according to $\lambda_{\text {blaze }}$. For this configuration, a maximum of performance is clearly obtained at $480 \mathrm{~nm}$. This wavelength will be chosen to design the height of the grating grooves.

\subsection{Theoretical concentrator performance and tolerances}

\subsubsection{Expected performance}

Theoretical results are obtained with hypotheses and means presented in 5.1, 5.2, 5.3. The full system is composed of 5 lenses and 5 grooves. It will be compared to experimental results in Section 6.5. Theoretical optical results are the following:

- A geometrical factor of $5 \times$ is reached.

- A theoretical optical efficiency is evaluated at $73 \%$.

- Spectral separation efficiency can be described by Fig. 12(a), with a grating period about $25 \mu \mathrm{m}$.

\subsubsection{System tolerancing}

Tolerance analysis is an important step before the manufacturing process, to compute expected performance degradation due to manufacturing imprecisions. The merit functions are fluxes collected on both cells normalized to their respective spectral domain of interest. Degradation is evaluated for several tolerance parameters individually, in order to isolate each effect.

From these analyses, a tolerance matrix is derived. It gives the values of each tolerance parameter that induces, for at least one of the merit functions, a decrease of $1 \%, 5 \%, 10 \%$, and $20 \%$. The tolerance analysis was also performed with ASAP $^{\circledR}$.

For a better understanding of the phenomenon, only the central lens was considered (but all grooves were considered). The tolerance parameters are translations and rotations of some parts of the system (waveguide, lens, grating, lens + grating). When considering rotations of an element, the rotation center is always the center of this element. A tolerance analysis is also performed on the tracking-error, which makes the sunlight obliquely incident on the system. Fig. 14(a), for example, shows the evolution of the merit function for a horizontal translation of the waveguide relative to the lens array set. When the departure from the ideal case is not too large, a larger proportion of the light gets to one side of the groove. Consequently, the flux on one detector decreases while the flux on the other detector increases. If the departure from the ideal case is too great, the light is no longer captured by the groove and thus the flux decreases on both detectors. Increasing the groove size doesn't improve the tolerance of the system because it is mainly the position of the $600 \mathrm{~nm}$ light beam intensity peak on the center of the groove that determines the spectral splitting efficiency. However, a 


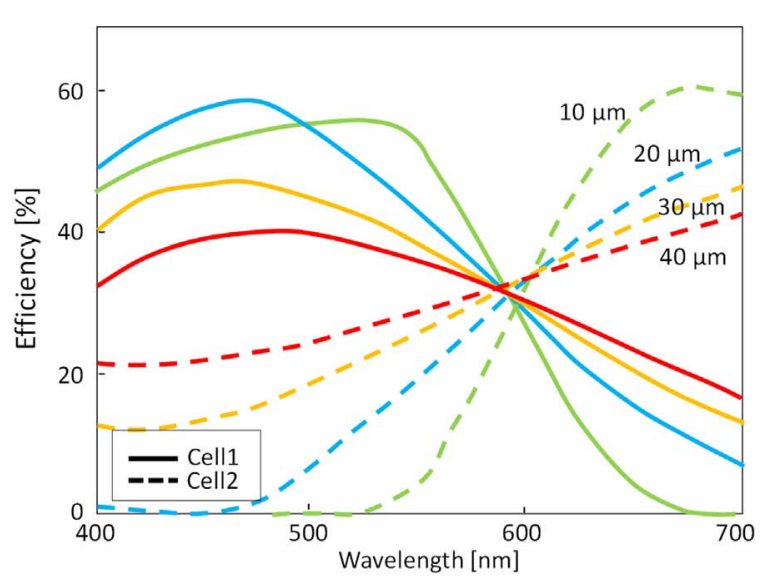

(a)

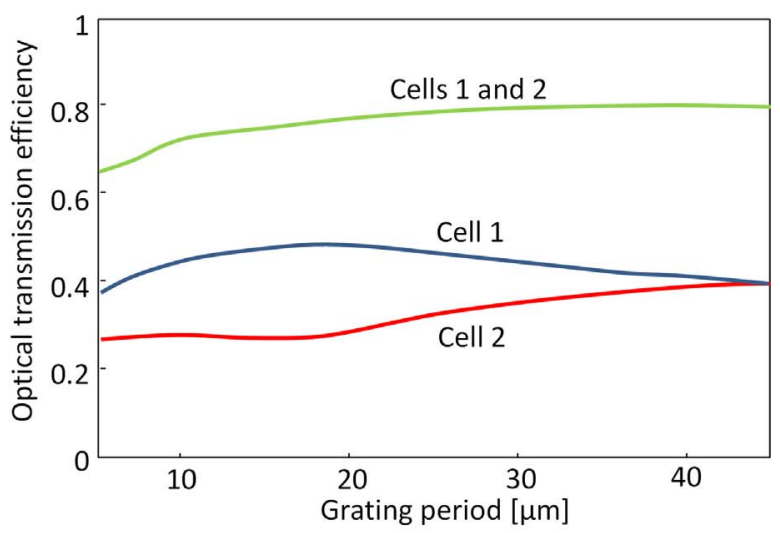

(b)

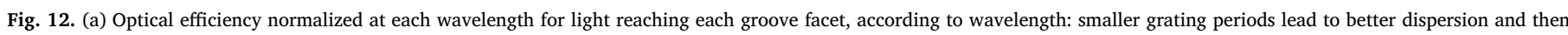

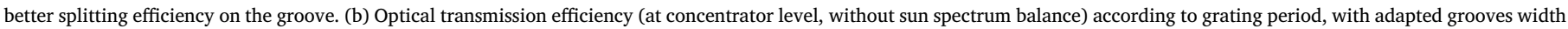
to collect all light of interest: from an optical transmission efficiency point of view, a $40 \mu \mathrm{m}$ grating period is the optimum.

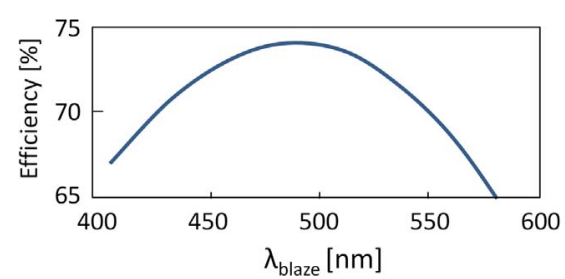

Fig. 13. Global system optical efficiency according to the blaze wavelength taking into account the sun spectrum, all parameters and optical elements: an optimum is found for $\lambda_{\text {blaze }}=480 \mathrm{~nm}$.

larger groove means the beam is captured by the groove until larger deviations from the ideal case occur, thus keeping a larger part of the incident flux on at least one of the detectors. This effect is visible in Fig. 14(a), where the merit function presents a peak for the smaller groove $(1 \mathrm{~mm})$, while a plateau is present for the larger groove $(2 \mathrm{~mm})$.

For the other tolerance parameters, the principle remains the same.

As stated, a groove thickness of $2 \mathrm{~mm}$ has been chosen. Indeed, the decoupling is reasonable and, in case the manufacturing errors are too large, it increases the chance of having a large flux on at least one detector. The tolerance matrix is presented in Table 2. It shows for example that the system is particularly sensitive to a horizontal or vertical translation of the waveguide, as well as to a rotation along $\mathrm{X}$ of any of the individual elements. This is logical, as translations and rotations along $\mathrm{X}$ directly deviate the converging point from the groove center. The system is not very sensitive to a rotation along $\mathrm{X}$ of the lens and the grating simultaneously. Indeed, the rotation along $\mathrm{X}$ of the lens deviates the beam in the opposite way compared to the grating, thus simultaneously they compensate each other. The system is less sensitive to rotations of the elements along Y or Z. This comes from the fact that the considered rotation center is in the middle of the rotated element, thus the rotation along $\mathrm{Y}$ and $\mathrm{Z}$ only deviate a part of the light from the center of the groove. If the rotation center changes, the impact would be larger.

The data in Table 2 are mostly non-critical for our manufacturing process. It nevertheless needs an accurate alignment between focusing elements and the waveguide grooves. For our prototype, rotational and translation adjustment possibilities between each lens and the waveguide are included.

\section{Experimental prototype}

\subsection{Solar cell manufacturing}

The DSSCs with N3 and SQ2 dyes are prepared according to previous published works (Thalluri et al., 2013, 2015). Photovoltaic current-voltage (I-V) performances were recorded by a class A solar simulator supplied by Newport Spectra Physics coupled with a Keithley 2400 Source meter under simulated 0.77 sun illumination (see Fig. 15). The active areas of solar cells were $0.2064 \mathrm{~cm}^{2}$. The external quantum efficiency (EQE) measurements were performed using a monochromator (Kratos model GM 252) and a dedicated lamp (Kratos model LH 151N). Standard EQE curves are depicted in Fig. 2.

However, manufactured cells seem quite unstable, especially SQ2 cells. Cell performance reproducibility and longevity, especially for a large DSSC area as needed for this concentrator, are still challenging. This point is extensively studied in the GREENMAT laboratory.

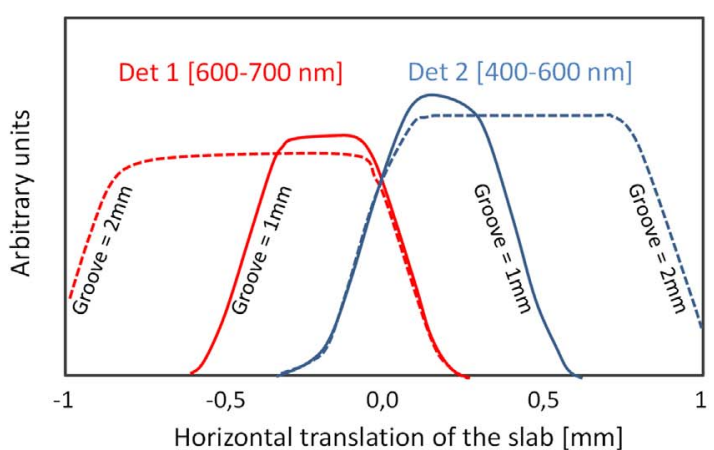

(a)
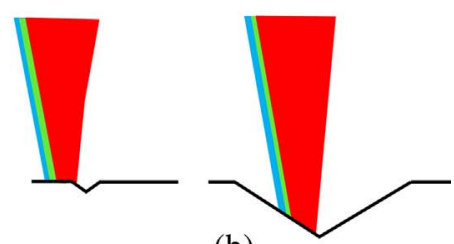

(b)
Fig. 14. (a) Merit function for a horizontal translation between the waveguide and the focusing element: a wider groove allows a broadening, but does not improve the sensitivity for small displacements. (b) Sketch of ray beam displacement on the groove due to a horizontal translation of $0.4 \mathrm{~mm}$, for two different groove widths. 
Table 2

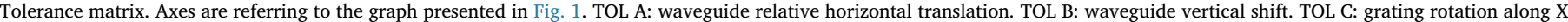

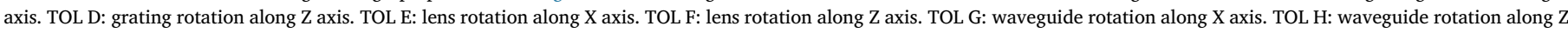
axis. TOL I: groove width modification ( $30^{\circ}$ facet inclination angle is not modified). TOL $\mathrm{J}$ : tracking error tolerance.

\begin{tabular}{|c|c|c|c|c|}
\hline Tolerance & $99 \%$ & $95 \%$ & $90 \%$ & $80 \%$ \\
\hline TOL A [mm] & \pm 0.002 & \pm 0.0098 & \pm 0.0197 & \pm 0.039 \\
\hline TOL B [mm] & \pm 0.0208 & \pm 0.1041 & \pm 0.2084 & \pm 0.4274 \\
\hline TOL C $\left[{ }^{\circ}\right]$ & \pm 0.0099 & \pm 0.0496 & \pm 0.0993 & \pm 0.1985 \\
\hline TOL D $\left[^{\circ}\right]$ & \pm 4.5867 & \pm 10.56 & \pm 15.0857 & $>20$ \\
\hline TOL E $\left[^{\circ}\right]$ & \pm 0.0092 & \pm 0.0458 & \pm 0.0916 & \pm 0.1831 \\
\hline TOL F $\left[^{\circ}\right]$ & \pm 2.29 & \pm 4.5425 & \pm 5.3315 & \pm 6.8198 \\
\hline TOL G $\left[{ }^{\circ}\right]$ & \pm 0.1041 & \pm 0.5206 & \pm 1.0382 & \pm 2.0036 \\
\hline TOL H $\left[{ }^{\circ}\right]$ & \pm 3.1689 & \pm 6.0963 & \pm 7.2677 & \pm 8.1968 \\
\hline TOL I [mm] & $\pm 0.5246 \leftrightarrow 0.9458$ & $<0.457 \leftrightarrow 1.24$ & $<0.475 \leftrightarrow 1.6458$ & $<0.475 \leftrightarrow 2.3922$ \\
\hline TOL J [ $\left.{ }^{\circ}\right]$ & \pm 0.0049 & \pm 0.0244 & \pm 0.0487 & \pm 0.0974 \\
\hline
\end{tabular}

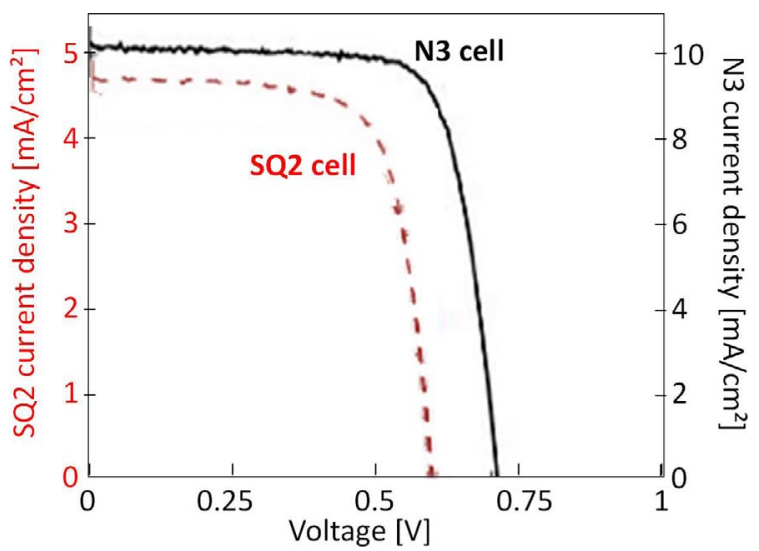

Fig. 15. IV curves of standard N3 and SQ2 solar cells (GREENMAT).

\subsection{Lenses and waveguide manufacturing}

To test the feasibility of our solar concentrator concept, a prototype has been manufactured and optically tested to compare theoretical and experimental results. Realizing such a prototype is not straightforward. Indeed, its manufacturing implies a lot of considerations other than the optical design. The fabrication process is detailed hereunder.

Lenses and waveguides were produced by injection molding of thermoplastic material (PMMA). This process is particularly relevant for low cost/high volume replication of polymer parts, including transparent optical elements with complex shapes and structures. The injection molding process consists in pushing PMMA granules in a chamber (barrel), where they are heated and sheared by a screw. The resulting molten material is then injected through a nozzle and a gate to fill a closed mold cavity. The shape of the cavity matches the geometry of the final part, except for shrinkage. The injected polymer is then cooled down before the mold is opened and the replicated part is ejected. Then the mold is closed again and a new molding cycle (generating a new replica) can start.

Mold inserts for the waveguide component were produced by Sirris in a tool steel material (reference TKM 2766 LBV) by combining wire electro-discharge machining (W-EDM, Fanuc Robocut 180iS-WB), micro-milling (Fanuc Robodrill 31-iMB5) and post-polishing (polishing flat bench - KEMET cube). Residual roughness was measured on the inserts by 3D optical profilometry (Sensofar Plu Neox) and values around $25 \mathrm{~nm}$ Ra down to $5 \mathrm{~nm}$ Ra were observed on the V-grooves and the main planar surfaces, respectively.

Refractive lenses were replicated from mold inserts made out of steel and MoldMax XL materials, for the flat and curved surfaces respectively. MoldMax material was pre-shaped by W-EDM and postmachined by ultra-precision diamond turning (MOORE Nanotech 350FG, by VUB/Belgium), while steel was processed according to the same procedure as for the waveguide. Roughness in the order of $5 \mathrm{~nm}$ Ra was also observed for these inserts.

Both optical components were replicated by injection molding with an Arburg Allrounder 420C equipment, based on a two-cavity mold, the first cavity for the waveguide and a second for the lens. PMMA material (SOLAR IM20 grade) was supplied by Evonik. The mold temperature was kept at $90{ }^{\circ} \mathrm{C}$ during the whole process. The injection time was more than $4 \mathrm{~s}$ and 1500 bar holding pressure was applied over more than $10 \mathrm{~s}$ per cycle, in order to promote highest shape conformity and lowest shrinkage.

The mold design was iteratively modified according to the molding constraints and the stringent optical requirements for the final parts. The injection gate particularly has been optimized, starting from a single-point then evolving towards a larger beam for the final components, aiming at promoting a good filling of the mold cavity, while reducing shrinking defects.

Final components exhibit nominal optical clarity, shape conformity and low roughness, in phase with the optical requirements for the foreseen application. The flat side of the lens is ready to receive its associated diffraction grating, thanks to a soft-UV-embossing process performed by CSL.

Finalized lens replicas are then mounted on a frame specifically developed by Sirris, allowing each lens element to be positioned precisely, one with respect to the other, prior to final fastening. Translation of the light guide is performed thanks to an electrically activated actuator (MP21, Micronix USA), in order to fine-tune the alignment of the lens stack with respect to the waveguide.

The final prototype is depicted in Figs. 16 and 17. More details can be found in Blain et al. (2014).
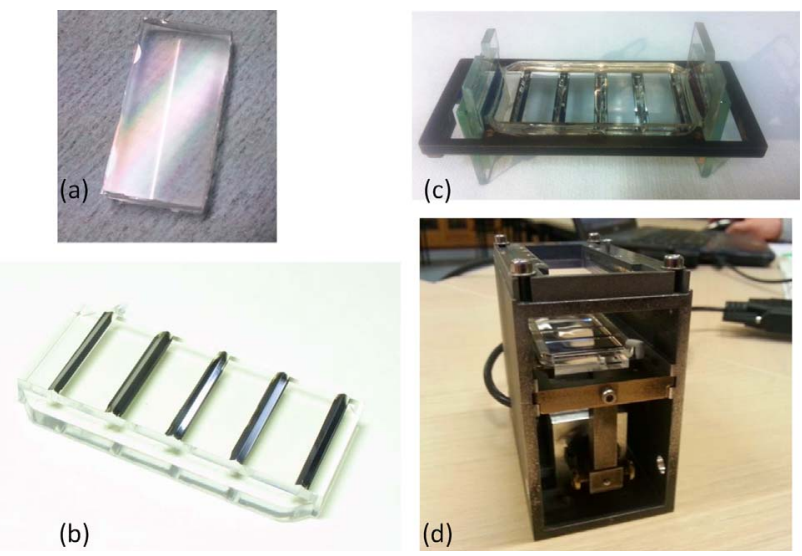

Fig. 16. Final prototype: (a) grating/lens $(22 \mu \mathrm{m}$ period grating imprinted in NOA76 on the injection processed PMMA lens from a diamond turned mold), (b) waveguide with aluminum coated grooves (in PMMA by injection process), (c) waveguide coupled with DSSC, (d) complete prototype without PV cells. It is composed of 5 grating/lenses above the waveguide. A motor allows an accurate alignment between the two optical elements. 

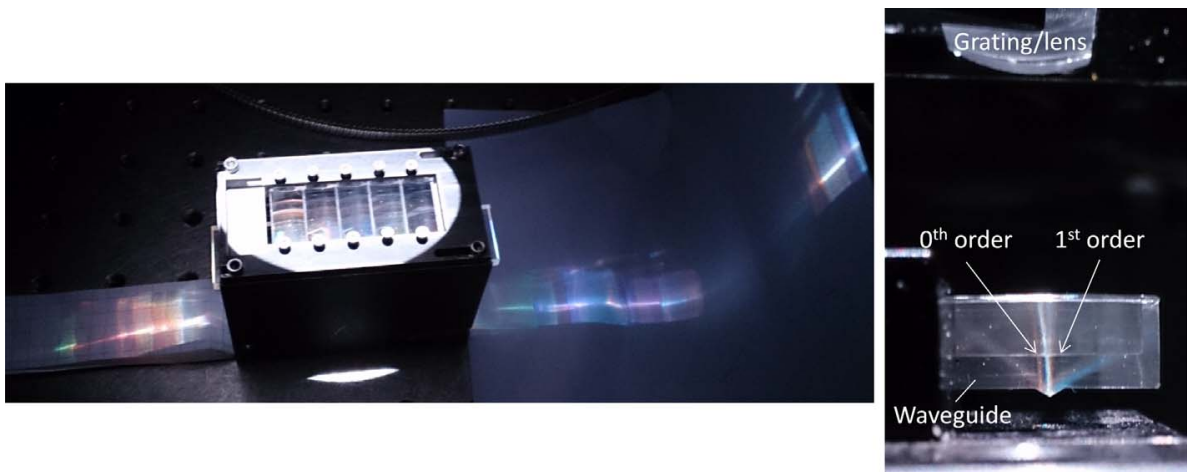

Fig. 17. Concentrator under the solar simulator light. Left image, the edges of the waveguide are not yet linked to PV cells, so spectral separation and light guiding to the edges is observable outside the waveguide: respectively, blue/red light mainly reaches the left/right edge. Right image, spectral separation is observable: light traveling through the waveguide, spatial dissociation between 0th and 1st diffraction orders, and finally spectral separation on the tip of the groove in the focal plane.

\subsection{Experimental set-up}

Experimental set-up and performances have been described extensively in Blain et al. (2014).

For indoor laboratory testing, the set-up includes:

- A collimated beam sun simulator (Thibert et al., 2010), developed by CSL: it is composed of a 750 W CERMAX ${ }^{\circledR}$ UV enhanced Xenon lamp and a beam shaping optical system which produces a collimated beam with a half-divergence of $\pm 0.25^{\circ}$ and an output of about $250 \mathrm{~W} / \mathrm{m}^{2}$. The beam offers a spatial uniformity of $90 \%$ over a $10 \times 10 \mathrm{~cm}^{2}$ area and $95 \%$ over a $6 \times 6 \mathrm{~cm}^{2}$ area. Its spectral irradiance is depicted in Fig. 18.

- A light sensing unit: (1) For lens/grating combination testing, this is composed of a 3 axis translation stage and a fiber coupled UV-vis spectrometer which will scan the focal plane. As the spectrometer is used, the spectrum is measured for each position of the fiber tip. Intensity maps can be computed by integration over the entire wavelength range of the spectrometer. (2) For full concentrator testing, small integrating spheres, fiber coupled with the UV-vis spectrometer, are placed at the edges of the waveguide.

- A tip/tilt adjustable mount to support the concentrator or part of it. A neutral density can be placed in the light path to avoid any sensor saturation.

\subsection{Sub-system characterization steps}

\subsubsection{Lens testing}

A lens is disposed on the mount. The flat face of the lens is facing the collimator and the convex face is toward the detector. Its origin position is known regarding the position of the sensor. The reference point is the geometrical center of the lens plane diopter. The distances and positions are evaluated regarding this reference point. For each lens, we explore the image space to localize the focal plane. The focal plane is considered at the maximum detected intensity. Once the focal spot is detected, the focal plane can be investigated (cf. Fig. 19). Measured focus in the air and offset from the center of the lens are in close agreement with the theoretical simulations: $\epsilon_{\text {focus }}<1 \%$ and $\epsilon_{\text {offset }}<1.4 \%$. This shows that the design and the manufacturing of the lens is correct and meets the objectives.

\subsubsection{Grating testing}

The grating to be imprinted on lenses is a replication of a commercial grating. ${ }^{1}$ Its replication fidelity has first been tested on a glass plate, through a diffraction efficiency characterization performed at CSL. Diffraction efficiency measurements are depicted in Fig. 20 at first diffraction order (the order that is relevant for the concentrator performance): results show close diffraction efficiency between the master

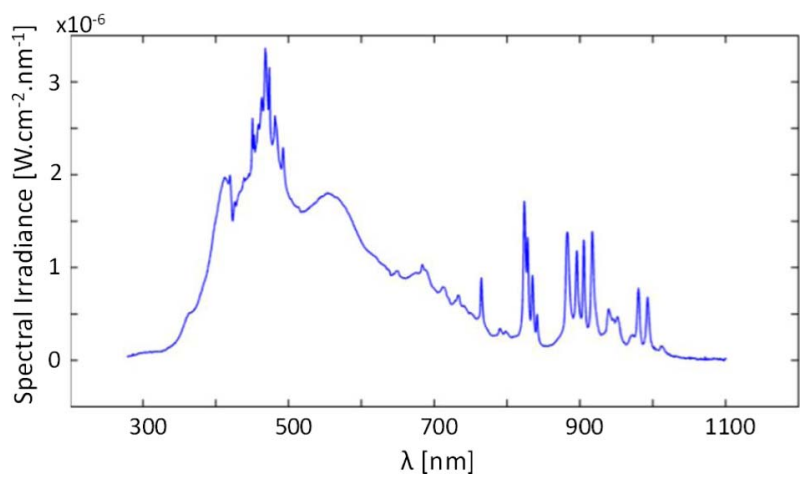

Fig. 18. Solar simulator spectral irradiance measured at CSL.

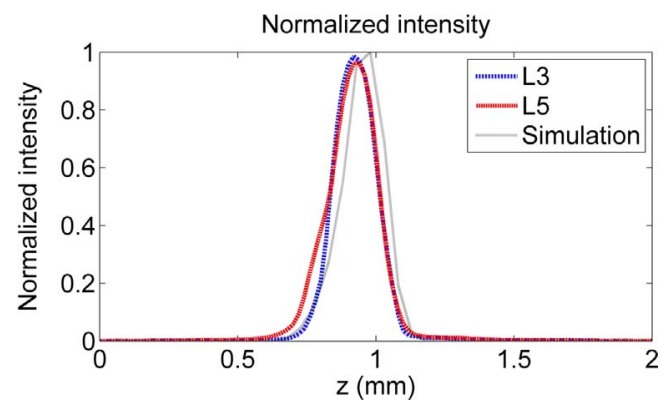

Fig. 19. Profile of the focal plane: Offset position of the focal line for two different lenses (L3 and L5) compared to the simulation.

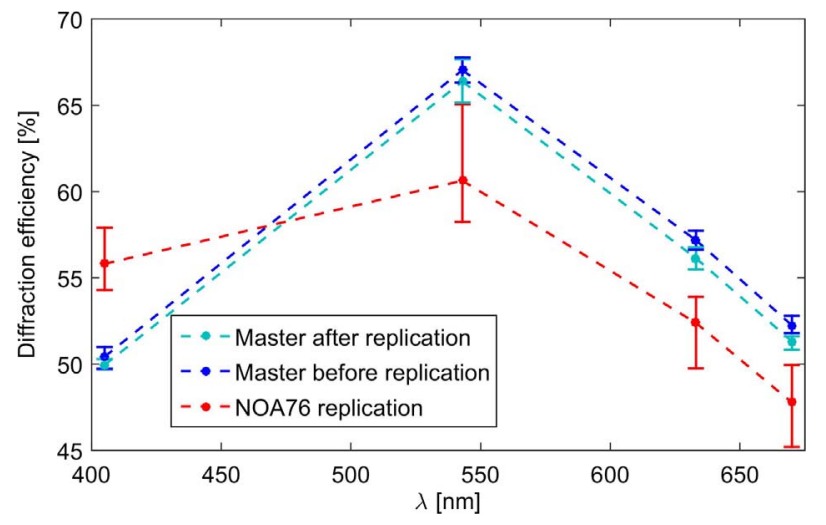

Fig. 20. Measured diffraction efficiencies of BK7 commercial grating, and CSL grating replications (NOA76) on glass plate. The small shift between maxima comes from the refractive index delta between materials. 


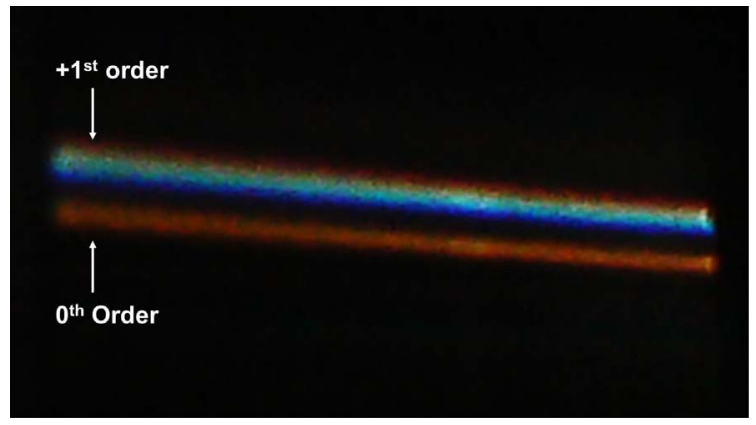

Fig. 21. Picture of the focal plane of a lens/grating combination. Splitting between 0 and 1 st diffraction orders and spectral spreading in the 1st diffraction order can be observed as expected.

and the replications, indicating a high quality replication process.

\subsubsection{Lens/grating combination testing}

The aim of this experiment is to characterize the position and the spectral spread of the 1st diffraction order in the focal plane. Here our reference position is the position of the 0th order. Fig. 21 shows a photograph of two lines corresponding to the 0th and the 1st diffraction orders. The spatial shift between the orders is directly observable and so is the spectral spread of the 1st order. The other diffracted orders are so weak that these are not seen on the picture.

With the same spectrometer mounted on a 3-axis translation stage as used in the previous section, the focal plane has been scanned. Some measurements are depicted in Fig. 22, for three different spectral ranges: the two spectral ranges of interest $(400-600 \mathrm{~nm}$ and $600-700 \mathrm{~nm}$ ) and at $1050 \mathrm{~nm}$ in the IR domain. The $1050 \mathrm{~nm}$ image has a low signal-to-noise ratio but it establishes which of the beams is the 0th diffraction order. Theoretically the distance between the two centers of the domains is $0.15 \mathrm{~mm}$. Experimentally, the mean distance is $0.15 \mathrm{~mm}$, with a standard deviation around $0.014 \mathrm{~mm}$. Concerning the distance between the 0th and 1st diffraction orders, the measurement is slightly smaller than expected $(0.03 \mathrm{~mm}$ closer). This might result in part from the 0th order being reflected by the groove, which is not a big issue (a small part of the IR light will be collected by the grooves and sent to a cell, slightly increasing the cell temperature).

\subsection{Global concentrator optical performance}

When coupling with the waveguide, the test set-up is slightly modified: at the waveguide output, the propagating angles are quite large (up to $\pm 45^{\circ}$ ). In order to collect and analyze all the output light, an integrating sphere is used. As a test, only one lens is placed in front of the waveguide. At the end, all lens positions have been individually

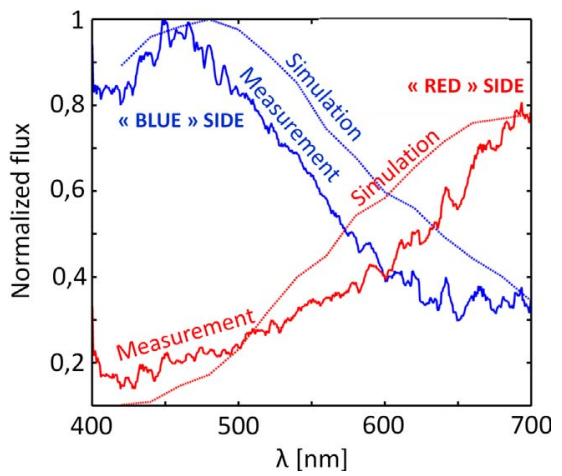

Fig. 23. Normalized spectral fluxes collected at the waveguide edges. "Blue" side: dedicated to reach N3 cell. "Red" side: dedicated to reach SQ2 cell. The experimental spectral separation is close to the theoretical curves (i.e. at $600 \mathrm{~nm}$ ). (For interpretation of the references to color in this figure legend, the reader is referred to the web version of this article.)

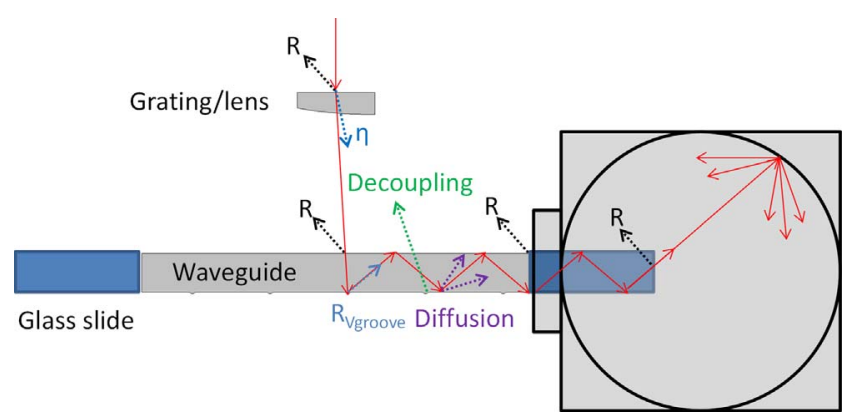

Fig. 24. Scheme of optical losses. Losses by reflection: $4 \%$. Losses by diffraction: $45 \%$. Losses due to imperfect reflection coating on waveguide grooves: $15 \%$. Losses by decoupling: $17.5 \%$. Losses by scattering: $3 \%$. Also some losses by vignetting. Global transmission reaches no more than $55 \%$.

tested. An image of the spectral separation achieved is depicted in Fig. 17, while normalized results are compared to theoretical curves in Fig. 23: results show spectral splitting behavior around $600 \mathrm{~nm}$, in accordance with theoretical simulations.

However, large optical losses occur, and optical transmission reaches no more than 55\%. The optical loss sources are depicted in Fig. 24.

\subsection{Experimental performance with cells}

The final concentrator is coupled with solar cells at the edges, and tested in front of the solar simulator. Results are depicted in Fig. 25. The design of the waveguide, as discussed so far, has been calculated to give
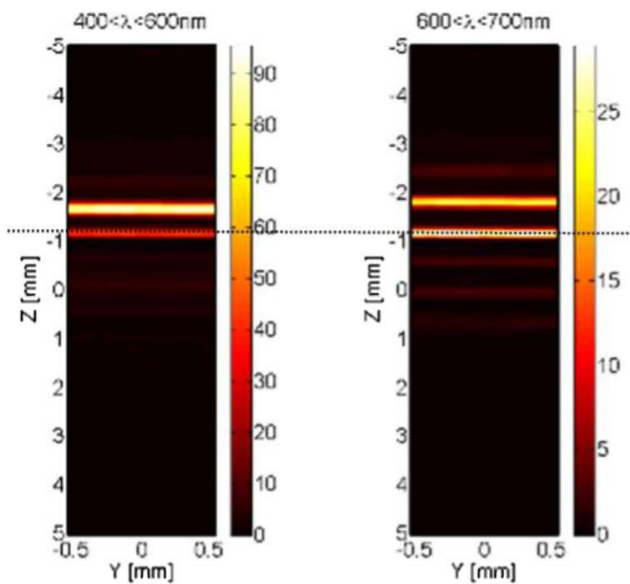

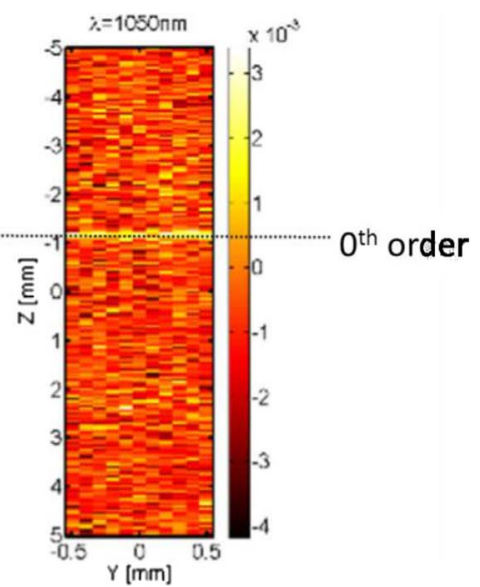

Fig. 22. From left to right: Intensity (percentage) in the focal plane of a lens/grating combination for wavelengths from 400 to $600 \mathrm{~nm}, 600$ to $700 \mathrm{~nm}$ and a wavelength of $1050 \mathrm{~nm}$. 


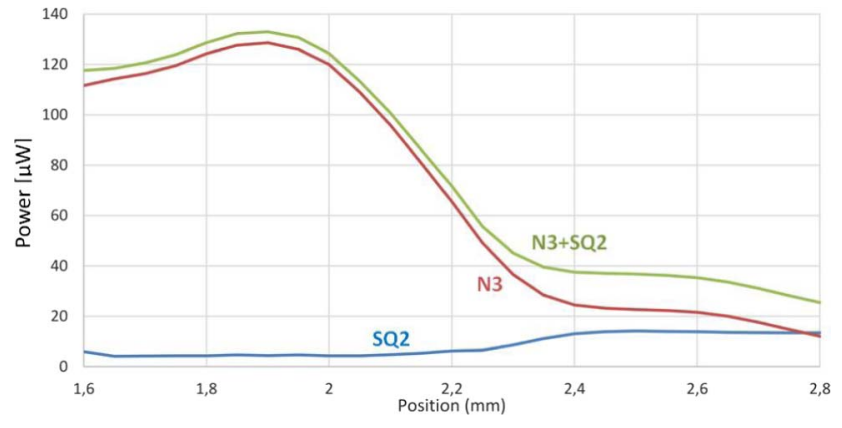

Fig. 25. Cell output power measured in front of a solar simulator (indoor test set-up with 5 lenses assembled), according to waveguide position. For position $=2.2 \mathrm{~mm}$, the waveguide is aligned with concentrator lenses with optimum spectral separation.

maximal performance at a position of $2.2 \mathrm{~mm}$, giving the strongest spectral separation for individual lighting of equally efficient solar cells with selective absorption ranges. However, due to their instability (as introduced in Section 6.1), SQ2 cells especially have very low performance. In this case, the experimental test shows that when one of the solar cells is working badly, the waveguide position can be adapted to partially compensate this loss and maximally exploit the actual performance of its components. This makes this device a flexible approach to selective wavelength light harvesting. This results is of course not representative of the expected concentrator electrical performance, but it is a promising functional test. Improvement of cell stability is in progress at GREENMAT.

\section{Conclusion}

A new concept of solar concentrator has been introduced. The optical concept is based on a waveguided solar concentrator with the addition of spectral splitting. The spectral splitting allows two cells to be used simultaneously with different operational spectra, intending to increase global efficiency. Coupling with waveguiding allows a concentration factor to be introduced, reducing cell area.

Optical design and its related optimization methodology were fully developed in this paper. Moreover a first functional prototype has been realized including every optical element and dye sensitized solar cell as an example. This concentrator prototype was characterized under laboratory conditions, with the main objective to understand how the concentrator works and what are its potential applications. The concentrator shows good theoretical results with $5 \times$ concentration ratio for $73 \%$ of theoretical optical efficiency. Experimental optical measurements were compared with theoretical simulations, with great agreement, except for some extra losses that were identified. While electrical testing was not representative, this study has shown that the proposed concentrator design is promising from a spectral splitting and concentration point of view. It is notable that the concept is of course adaptable to any combination of PV cells. It needs only minor adaptation for other spectral ranges to be collected at the edges according to chosen cells.

\section{Acknowledgments}

The authors gratefully acknowledge the Walloon Region for the grant accorded to the Guide2Dye project (convention $n^{\circ} 1117491$ ).

\section{References}

Anton Paar. Abbemat mw multiwavelengths refractometer. < http://www.anton-paar. com/be-en/products/details/multiwavelengths-refractometer-abbemat-wr-mw/ > (Accessed: 2016-03-07).

Blain, P., Michel, C., Clermont, L., Languy, F., Dcultot, M., Habraken, S., Lenaerts, C., Fleury-Frenette, K., Vandormael, D., Loicq, J., 2014. Spectral splitting planar solar concentrator: experimental testing of a design aiming at dye sensitized solar cells.
Proc, SPIE 9140, 91400S.

Chong, K., Siaw, F., Wong, C., Wong, G., 2009. Design and construction of non-imaging planar concentrator for concentrator photovoltaic system. Renew. Energy 34, 1364.

Coventry, J.S., 2005. Performance of a concentrating photovoltaic/thermal solar collector. Solar Energy 78, 211.

Cvetković, A., Hernández, M., Benítez, P., Miñano, J.C., Mohedano, R., Santamaría, A., 2007. The xr nonimaging photovoltaic concentrator. In: International Workshop on Concentrating PV.

Davis, A., 2009. Raytrace assisted analytical formulation of fresnel lens transmission eciency. In: Proceedings of SPIE 7429, 74290D.

Evonik Performance Materials GmbH. Plexiglas solar im20. < http://www.plexiglaspolymers.com/sites/dc/Downloadcenter/Evonik/Product/PLEXIGLAS-MoldingCompounds/en/Product-Information/PLEXIGLAS-Solar-IM20-E.pdf > (Accessed: 2016-03-07).

Ferrer-Rodrguez, P.J., Baig, H., Fernndez, E.F., Almonacid, F., Mallick, T., Prez-Higueras, P., 2017. Optical modeling of four fresnel-based high-cpv units. Solar Energy 115, 805.

Feuermann, D., Gordon, J.M., 2001. High-concentration photovoltaic designs based on miniature parabolic dishes. Solar Energy 5, 423.

Fisher, B., Biddle, J., 2011. Luminescent spectral splitting: efficient spatial division of solar spectrum at low concentration. Solar Energy Mater. Solar Cells 95 (7), 1741-1755.

Fixler, Bar-David, N., Zalevsk, Z., 2011. Spectral separation of sunlight for enhanced operability of photovoltaic cells. J. Photon. Energy 1.

Gallagher, S., Norton, B., Eames, P., 2007. Quantum dot solar concentrators: electrical conversion efficiencies and comparative concentrating factors of fabricated devices. Solar Energy 81, 813.

Goldschmidt, J.C., Peters, M., Bösch, A., Helmers, H., Dimroth, F., Glunz, S.W., Willeke, G., 2009a. Increasing the efficiency of fluorescent concentrator systems. Solar Energy Mater. Solar Cells 93, 176.

Goldschmidt, J.C., Peters, M., Hermle, M., Glunz, S.W., 2009b. Characterizing the light guiding of fluorescent concentrators. J. Appl. Phys. 105, 1-9.

Goodman, J.W., 1968. Introduction to Fourier Optics. The McGraw-Hill Companies, Inc.

Helmers, H., Thor, W., Schmidt, T., van Rooyen, D., Bett, A., 2013. Optical analysis of deviations in a concentrating photovoltaics central receiver system with a flux homogenizer. Appl. Opt. 52, 2974-2984.

Karp, J.H., Tremblay, E.J., Ford, J.E., 2010. Planar micro-optic solar concentrator. Opt. Exp. 18 (2), 1122-1133.

Karp, J.H., Tremblay, E.J., Hallas, J.M., Ford, J.E., 2011. Orthogonal and secondary concentration in planar micro-optic solar collectors. Opt. Exp. 19 (S4), A673-A685.

Languy, F., Lenaerts, C., Loicq, J., Habraken, S., 2009. Achromatization of solar concentrator thanks to diffractive optics. In: 2nd International Workshop on Concentrating Photovoltaic Optics and Power, vol. 2.

Leutz, R., 2000. Nonimaging Fresnel Lenses for Solar Concentrators. Ph.d. thesis. Tokyo University of Agriculture and Technology.

Li, X., Wu, W.-X., Zhang, C.-M., Zhang, A., Luo, Y.-P., Zhang, Z.-W., Deng, R.-J., Zou, C., Meng, T., 2016. Fabrication and characterization of planar luminescent solar concentrator waveguides based on lr305 dye. Spectroscopy Spectral Anal. 36 (4).

Luque, A.L., Andreev, V.M., 2007. Concentrator Photovoltaics. Springer-Verlag, New York, LLC.

McConnell, R., Symko-Davies, M., 2006. Multi junction photovoltaic technologies for high-performance concentrators. In: WCPEC-4, vol. NREL/CP-520-39791.

Michel, C., Loicq, J., Languy, F., Habraken, S., 2014. Optical study of a solar concentrator for space applications based on a diffractive/refractive optical combination. Solar Energy Mater. Solar Cells 120, 183-190.

Michel, C., Loicq, J., Thibert, T., Habraken, S., 2015. Optical study of diffraction grating/ fresnel lens combinations applied to a spectral-splitting solar concentrator for space applications. Appl. Opt. 54 (22).

Ming-Chin, C., Tung, Y.L., Tien, C.-H., 2009. Ultracompact backlight-reversed concentration optics. Appl. Opt. 48 (21), 4142-4148.

Mojiri, A., Taylor, R., Thomsen, E., Rosengarten, G., 2013. Spectral beam splitting for efficient conversion of solar energy - a review. Renew. Sust. Energy Rev. 28, 654-663.

National Renewable Energy Laboratory. Reference solar spectral irradiance: Astm g-173. < http://rredc.nrel.gov/solar/spectra/am1.5/ASTMG173/ASTMG173. html > (Accessed: 2015).

Norland Products. Norland optical adhesive 76. < https://www.norlandprod.com/ adhesives/noa\%2076.html > (Accessed: 2016-08-11).

O'Gallagher, J.J., 2008. Nonimaging Optics in Solar Energy. Morgan and Claypool Publishers.

Park, M., Oh, K., Kim, J., Shin, H.W., Oh, B.D., 2010. A tapered dielectric waveguide solar concentrator for a compound semiconductor photovoltaic cell. Opt. Exp. 18 (2), 1777-1787.

Peng, X., Lin, H., Liu, Y., Li, B., 2014. Total internal reflection-based planar waveguide solar concentrator with symmetric air prisms as couplers. Opt. Exp. 22 (S6), A1389.

Pindar, A., Marcroft, D., Nichols, A., 1992. United States Patent 2 (12).

Stefancich, M., Zayan, A., Chiesa, M., Rampino, S., Roncati, D., Kimerling, L., Michel, J., 2012. Single element spectral splitting solar concentrator for multiple cells cpv system. Opt. Exp. 20 (8), 9004.

Steiner, M., Gerstmaier, T., Bett, A., 2016. Concentrating photovoltaic systems. In: The Performance of Photovoltaic (PV) Systems: Modelling, Measurement and Assessment, pp. 297-320.

Swanson, G.J., 1989. Binary Optics Technology: The Theory and Design of Multi-level Diffractive Optical Elements, vol. 854 Massachusetts Institute of Technology, Cambridge.

Takamoto, T., Kaneiwa, M., Imaizumi, M., Yamaguchi, M., 2005. Ingap/gaas-based multi 
junction solar cells. Prog. Photovolt.: Res. Appl. 13, 495.

Thalluri, G.K.V.V., Décultot, M., Henrist, C., Dewalque, J., Colson, P., Habraken, S., Spoltore, D., Manca, J., Cloots, R., 2013. Morphological and opto-electrical properties of a solution deposited platinum counter electrode for low cost dye sensitized solar cells. Phys. Chem. Chem. Phys. 15 (45), 19799-19806.

Thalluri, G.K.V.V., Henrist, C., Spronck, G., Vertruyen, B., Dewalque, J., Colson, P., Cloots, S.H.R., 2015. Elucidating the opto-electrical properties of solid and hollow titania scattering layers for improvement of dye-sensitized solar cells. Thin Solid Films 594, 115-119.

Thibert, T., Hellin, M., Loicq, J., Mazy, E., Gillis, J., Languy, F., Habraken, S., Lecat, J., Defise, J., 2010. Continuous solar simulator for concentrator photovoltaic systems. In: 25th European Photovoltaic Solar Energy Conference, Spain.

van Sark, W., Barnham, K.J., Slooff, L., Chatten, A., Bchtemann, A., Meyer, A.,

McCormack, S., Koole, R., Farrell, D., Bose, R., Bende, E., Burgers, A.R., Budel, T.,
Quilitz, J., Kennedy, M., Meyer, T., Doneg, C.M., Meijerink, A., Vanmaekelbergh, D., 2008. Luminescent solar concentrators - a review of recent results. Opt. Exp. 16 (26), 21773-21792.

Vasylyev, S., 2005. Nonimaging reflective lens concentrator. In: International Conf. on Solar Concentrators for the Generation of Electricity or Hydrogen, Scottsdale.

Vlasov, A., Khvostikov, V., Karlina, L.B., Sorokina, S., Potapovich, N., Shvarts, M.,

Timoshina, N., 2013. Spectral-splitting concentrator photovoltaic modules based on algaas/gaas/gasb and gainp/ingaas(p) solar cells. Techn. Phys. 58 (7), 1034-1038. Wurfel, P., 2005. Physics of Solar Cells: From Principles to New Concepts. Wiley-VCH. Xiea, W., Daia, Y., Wanga, R., Sumath, K., 2011. Concentrated solar energy applications using fresnel lenses: a review. Renew. Sust. Energy Rev. 15, 2588-2606.

Zhao, L., 2011. High Efficiency Mechanically Stacked Multi-junction Solar Cells for Concentrator Photovoltaics. Ph.d. thesis, Katholieke Universiteit Leuven in collaboration with IMEC. 